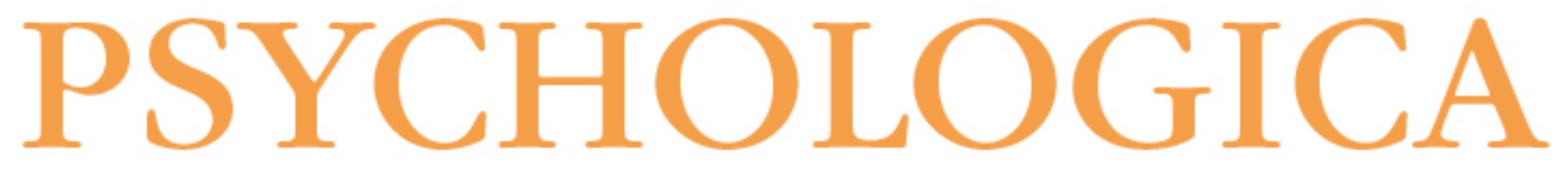

\title{
Necessidades dos familiares de doentes terminais em cuidados paliativos: revisão
} crítica da literatura
Autor(es):
Areia, Neide P.; Major, Sofia; Relvas, Ana Paula
Publicado por: Imprensa da Universidade de Coimbra
URL
persistente:
URI:http://hdl.handle.net/10316.2/42382
DOI:
DOI:https://doi.org/10.14195/1647-8606_60-1_8
Accessed : $\quad$ 26-Apr-2023 13:54:01

A navegação consulta e descarregamento dos títulos inseridos nas Bibliotecas Digitais UC Digitalis, UC Pombalina e UC Impactum, pressupõem a aceitação plena e sem reservas dos Termos e Condições de Uso destas Bibliotecas Digitais, disponíveis em https://digitalis.uc.pt/pt-pt/termos.

Conforme exposto nos referidos Termos e Condições de Uso, o descarregamento de títulos de acesso restrito requer uma licença válida de autorização devendo o utilizador aceder ao(s) documento(s) a partir de um endereço de IP da instituição detentora da supramencionada licença.

Ao utilizador é apenas permitido o descarregamento para uso pessoal, pelo que o emprego do(s) título(s) descarregado(s) para outro fim, designadamente comercial, carece de autorização do respetivo autor ou editor da obra.

Na medida em que todas as obras da UC Digitalis se encontram protegidas pelo Código do Direito de Autor e Direitos Conexos e demais legislação aplicável, toda a cópia, parcial ou total, deste documento, nos casos em que é legalmente admitida, deverá conter ou fazer-se acompanhar por este aviso.

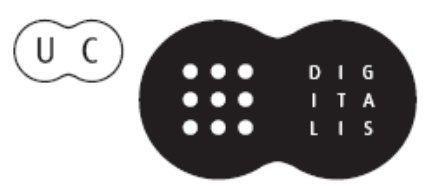


vOLUME
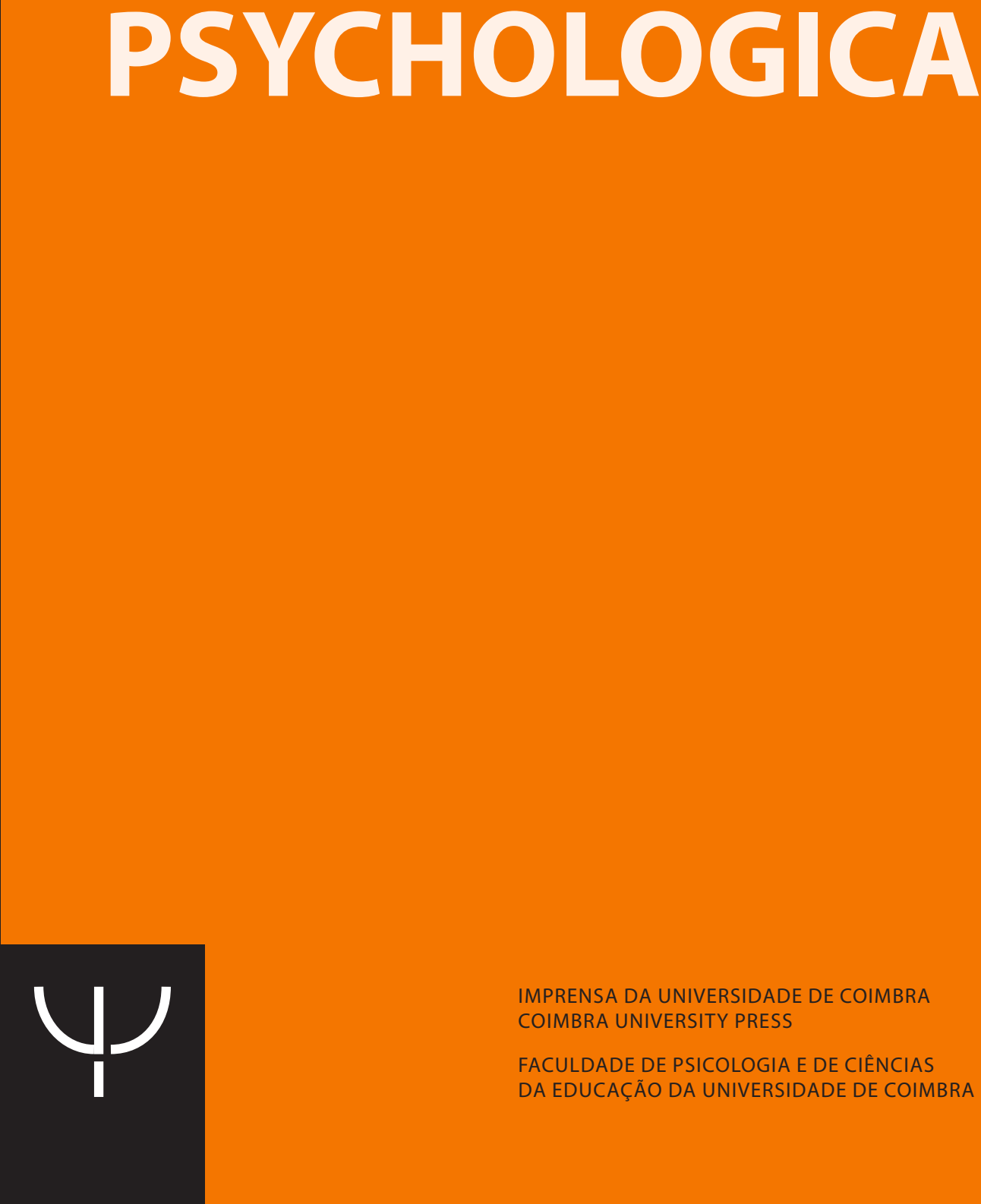

IMPRENSA DA UNIVERSIDADE DE COIMBRA

COIMBRA UNIVERSITY PRESS

FACULDADE DE PSICOLOGIA E DE CIÊNCIAS

DA EDUCAÇÃO DA UNIVERSIDADE DE COIMBRA 


\title{
Necessidades dos familiares de doentes terminais em cuidados paliativos: Revisão crítica da literatura
}

\author{
Neide P. Areia ${ }^{1}$, Sofia Major ${ }^{2}$ e Ana Paula Relvas ${ }^{3}$
}

Needs of the terminally ill's relatives in palliative care: Critical review of literature

\begin{abstract}
Terminal illness and death are critical events that interfere in the family system. The knowledge about illness and death's impact on the family is, still, scarce. Therefore, a critical review of literature was conducted with the general goal of determining the impact of terminal illness on the family and which are the family's support needs. The findings suggest that terminal illness is one of the most difficult experiences for the family system and an inadequate or non-existent approach to the family's problems can put that system in risk. Therefore, palliative care aims at the integration of the family in the care plan. However, the empirical production about this topic is insufficient, which compromises the development of new efficient family approaches that are sensitive to family needs. By this way, it is important to have more research that aims to determine the family needs of terminally ill patients and develop new family intervention strategies.
\end{abstract}

Keywords: terminal illness; family; grief; palliative care; family intervention

1 Bolseira de Doutoramento FCT: SFRH/BD/86178/2012, Faculdade de Psicologia e de Ciências da Educação, Universidade de Coimbra. Email: neideareia@hotmail.com

2 Professora Auxiliar Convidada da Universidade da Beira Interior, Departamento de Psicologia e Educação. Email: smajor@fpce.uc.pt

3 Professora Catedrática da Faculdade de Psicologia e de Ciências da Educação, Universidade de Coimbra. Email: aprelvas@fpce.uc.pt

Artigo recebido a 17-04-2016 e aprovado a 01-08-2016. 


\section{Resumo}

A doença terminal e a morte são acontecimentos críticos que interferem no sistema familiar. Considerando a escassez de literatura sobre o assunto, procedeu-se a uma revisão crítica da literatura, com o objetivo geral de determinar o impacto que a doença terminal tem na família e quais as suas necessidades de suporte. Concluiu-se que a doença terminal constitui uma das experiências mais difíceis para o sistema familiar e que uma abordagem formal inadequada dos problemas emergentes o pode colocar em risco. Por isto, os cuidados paliativos visam a integração da família no plano de cuidados. Todavia, verificou-se que a produção empírica neste âmbito é insuficiente, comprometendo o desenvolvimento de modalidades de intervenção eficazes e sensíveis às necessidades que emergem na família. Urge desenvolver mais estudos que visem determinar as necessidades das famílias do doente terminal e desenvolver estratégias de intervenção dirigidas a essas mesmas famílias.

Palavras-chave: doença terminal; família; luto antecipatório; cuidados paliativos; intervenção familiar

\section{INTRODUÇÃO}

A fase terminal de uma doença constitui um dos períodos mais difíceis para o doente e sua família. O acompanhamento da degradação física de um familiar e a eminência da sua morte podem constituir uma experiência altamente traumatizante e negativa para os sistemas familiares (Pereira, 2010), que importa conhecer de forma aprofundada. Apesar de ser amplamente aceite a importância crítica das famílias no acompanhamento ao doente em fim de vida, pouco se sabe sobre o impacto que a doença terminal tem no sistema familiar e nos seus elementos. Por esta razão, existem muito poucas diretrizes no que diz respeito à melhor forma de intervir e oferecer suporte às famílias. Como tal, preconiza-se a necessidade de se implementarem mais estudos empíricos que visem determinar a influência da doença terminal nas famílias, as suas necessidades de suporte, bem como as estratégias de intervenção que melhor lhes possam responder (Given, Given, \& Sherwood, 2012).

A presente revisão crítica de literatura enquadra-se no âmbito do reconhecimento da escassez de estudos empíricos nesta área (Carolan, Smith, \& Forbat, 2015) e tem como objetivos: a) determinar o impacto que o acompanhamento ao doente em fase terminal tem na dinâmica e funcionamento do sistema familiar, seus elementos, de um modo geral, e no cuidador principal, em particular; b) compreender como é experienciado o luto antecipatório no contexto familiar; c) esclarecer como os 
cuidados paliativos, enquanto abordagem holística, podem constituir uma resposta ao doente em fim de vida e sua família; e d) determinar quais as abordagens psicoterapêuticas sistémicas, devidamente validadas, que respondem eficazmente aos problemas emergentes, nesta fase, no sistema familiar.

Para tal, procedeu-se a uma revisão crítica da literatura, onde foram privilegiados artigos científicos publicados na última década (2006 - 2016) e livros na área da psicologia da doença crónica, família e cuidados em fim de vida. Incluíram-se, ainda, trabalhos anteriores ao período de referência, quando considerados determinantes para a compreensão da temática em estudo, particularmente os modelos teóricos e de intervenção alicerçados numa perspetiva Sistémica (e.g., Paradigma Normativo Sistémico da Doença Crónica, Terapia Familiar Focada no Luto). Para a pesquisa de artigos recorreu-se a bases de dados online -, B-On, EBSCO e ProQuest - utilizando termos de pesquisa e respetivas variações como "doença terminal", "cuidados em fim de vida" e "cuidados paliativos", combinados com termos como "família"; "funcionamento familiar" e "cuidadores". As publicações incluídas neste estudo foram selecionadas com base no seu conteúdo (e.g., objetivos dos estudos empíricos congruentes com os objetivos preconizados para este estudo de revisão de literatura) e através de uma leitura preliminar do resumo que nos permitiu determinar a adequabilidade e interesse do estudo para a presente revisão de literatura.

Para a apresentação dos resultados, começamos por caracterizar a doença na fase terminal e agónica. Acreditamos que este breve enquadramento é relevante para a compreensão das exigências que a doença terminal impõe ao sistema familiar e, subsequente experiência de luto antecipatório, tópicos que abordamos posteriormente. Por se constituir um tema emergente na literatura, daremos, posteriormente, destaque ao familiar cuidador principal e às suas necessidades. Finalmente, apresentamos e detalhamos os objetivos dos cuidados paliativos no cuidado ao binómio doente-família. Neste ponto, faremos alusão a um programa de intervenção familiar sistémico, que se tem revelado eficaz no suporte às famílias que enfrentam problemas decorrentes da eminência da morte de um elemento do sistema.

\section{A DOENÇA NA FASE TERMINAL}

Considera-se que o doente entra na fase terminal quando: a) existe um diagnóstico de uma doença avançada, progressiva e incurável; b) quando todas as abordagens terapêuticas, anteriormente experimentadas, falharam na reversão do curso da doença; c) o doente experimenta sintomas (ou alterações de) ou outros problemas severos, com origem multifatorial; d) existe um importante impacto 
emocional no doente, família e profissionais de saúde, decorrente da aproximação, implícita ou explícita, da morte e; e) existe um prognóstico de vida inferior a seis meses (Sociedad Española de Cuidados Paliativos [SECPAL], s.d.).

A fase terminal da doença é marcada pela acentuada deterioração física do doente, acompanhada de um sofrimento multidimensional (e.g., físico, psicológico, espiritual) intenso. Podem surgir, nesta fase, novos sintomas ou agravamento de sintomas preexistentes. São, em geral, altamente complexos, difíceis de gerir e têm repercussões diretas na qualidade de vida do doente e seus familiares. Os doentes podem apresentar sintomatologia álgica (dor) moderada a severa, complicações no sistema respiratório (e.g., dispneia, estridor), gastrointestinal (e.g., náuseas, vómitos, obstipação), neurológico (e.g., delirium terminal) e urinário (e.g., incontinência urinária). Podem, ainda, apresentar-se asténicos, prostrados ou semicomatosos, desenvolver anorexia e/ou caquexia, perder o apetite e/ou a capacidade de deglutir. Ao nível emocional, os doentes podem apresentar níveis clinicamente significativos de ansiedade, depressão e síndrome de desmoralização (Bausewein et al., 2015).

A fase agónica ou agonia integra a fase terminal da doença e corresponde ao período em que se verifica o declínio das funções vitais do doente, culmina com a sua morte em horas ou dias, muitas vezes, em menos de 48 horas. Em agonia, muitos doentes apresentam-se asténicos, acamados, semicomatosos e apresentam sintomas como o delirium, dor, estridor, febre, dispneia, hipersudorese, incontinência urinária, náuseas e vómitos, hemorragias, retenção urinária, mioclonias e convulsões (Gonçalves, Alvarenga, \& Silva, 2003).

\section{A FAMÍLIA DO DOENTE TERMINAL}

A morte (e o morrer) não é um acontecimento individual ou restrito, mas de significado e impacto coletivo (Osswald, 2013), constituindo-se, muitas vezes, um dos maiores desafios da vida familiar (Rolland, 2005). Não basta, por isso, que o foco da intervenção assente no controlo da sintomatologia. Deve, de igual modo, atender às dificuldades decorrentes da doença, dos indivíduos nela envolvidos (e.g., doente, família e restante rede) e aos desafios psicossociais que se lhes impõem (Irwin \& Ferris, 2008; Rolland, 2005).

No Paradigma Normativo Sistémico da Doença Crónica, Rolland (2005) preconiza que na fase terminal da doença, o doente teme morrer antes de conseguir concretizar o seu "projeto de vida", ao mesmo tempo que teme morrer sozinho ou em sofrimento. Com o aproximar da morte, doente e familiares experimentam, de forma mais intensa, um luto antecipatório. Os familiares podem sentir-se ambivalentes entre o desejo de 
maior intimidade com o doente ou maior distanciamento. É comum a "conspiração do silêncio", em que ninguém fala da morte, apesar da sua presença constante no pensamento de cada um dos membros da família.

Para Monroe e Oliviere (2009, p. 4), “a crise da morte eminente é a última ameaça que se impõe ao sistema familiar", o que naturalmente impele o sistema para um estado de desequilíbrio. Os autores destacam, no seu trabalho, alguns temas e desafios que surgem no sistema familiar na fase terminal da doença. São eles: a conspiração do silêncio, a negação, o isolamento, a procura de um bode-expiatório, o distanciamento intrafamiliar, a emergência de segredos familiares, a raiva e o conflito, e o abuso ou negligência. Os autores atendem, ainda, às perdas anteriores e às variações culturais enquanto variáveis influentes na resposta da família à doença e morte (Monroe \& Oliviere, 2009).

Num estudo conduzido por Forbat, McManus e Haraldsdottir (2012), os autores confirmaram que à medida que as exigências na prestação de cuidados ao doente terminal aumentam e se complexificam, as relações no seio da família alteram-se, bem como as rotinas diárias de cada um dos seus membros. Nesta fase, dificuldades relacionais tendem a ser exacerbadas quer entre doente e cuidador(es), quer entre os restantes elementos da família. Por outro lado, relações recentes (e.g., formação do casal) correm risco de rutura. O controlo de sintomas mais complexos pode potenciar conflitos no seio da família. O cuidador principal informal tende a assumir o seu papel de prestação de cuidados, não só junto do doente, mas também de outros membros da família, sempre com a finalidade de os proteger dos desafios impostos pela doença. Finalmente, familiares e doente tendem a ser cautelosos na forma de comunicar, evitando falar sobre a progressão da doença, prognóstico e sentimentos.

Estudos recentes demonstram, ainda, que os familiares tendem a experimentar reações emocionais complexas ante a proximidade da morte que, quando transitórias, são esperadas (e.g., distress, perceção de uma diminuição de qualidade de vida) (Totman, Pistrang, Smith, Hennessey, \& Martin, 2015; Wadhwa et al., 2013). No entanto, e apesar de adaptativas, as respostas emocionais dos familiares devem ser formalmente monitorizadas com o objetivo de prevenir o desenvolvimento de respostas desajustadas, já que os familiares de doentes terminais correm o risco de desenvolver uma franca morbilidade psiquiátrica (e.g., ansiedade, depressão) (Askari, Madgaonkar, \& Rowell, 2012; Totman et al., 2015).

Por outro lado, face à doença terminal, famílias resilientes tendem a adaptar-se funcional e positivamente à crise da morte eminente, não só recuperando, mas também, crescendo com esta experiência. O sistema de crenças da família (e.g., atribuição de significado à doença e morte), a organização familiar (e.g., flexibilidade) e a comunicação (e.g., partilha aberta de emoções) são consideradas variáveis familiares fundamentais à adaptação funcional e ao crescimento familiar face à adversidade e/ou 
situação de stress (e.g., doença terminal) (Walsh, 2002, 2003). Por isto, muitas famílias entendem a morte como uma oportunidade para valorizar e perspetivar a vida (Walsh, 2002, 2003), ao redefinirem prioridades, investirem nas relações mais significativas e aproveitarem oportunidades. A morte, ou a sua eminência, consciencializa os seus indivíduos para a fragilidade e o valor da vida, incitando à resolução de assuntos e conflitos inacabados e ao investimento nas relações (Rolland, 2003). Deste modo, a doença terminal e a morte podem conferir ao sistema familiar uma oportunidade de crescimento, fortalecimento e de aquisição de novos recursos, eventualmente úteis em futuros desafios e/ou crises que o sistema venha a enfrentar (Walsh, 2003).

No entanto, a fase terminal de uma doença crónica é, indubitavelmente, uma das mais difíceis para todo o sistema familiar. A aceitação da inevitabilidade e aproximação da morte constitui-se altamente difícil e até mesmo inexequível em algumas famílias. A família, ao vivenciar os problemas decorrentes da fase terminal da doença e ao tornar-se disponível a prestar assistência e cuidados a um dos seus membros, passa por um processo complexo e desgastante acompanhado por um intenso sofrimento (Pereira, 2010).

\section{LUTO ANTECIPATÓRIO NA FAMÍLIA}

A morte ou a sua eminência afeta todo o sistema familiar, envolvendo múltiplas perdas, não só de um membro do sistema, mas também de papéis e relações, de uma unidade familiar intacta, bem como o desaparecimento de esperanças, sonhos e projetos para todos os membros da família. Por isto, é o desafio mais difícil que o sistema familiar deve enfrentar, constituindo-se um fenómeno multidimensional, relacional e, ao mesmo tempo, individual, em que cada elemento da família experimenta o seu próprio luto e a sua resposta influi, necessariamente, na da do sistema e vice-versa (Kissane \& Bloch, 2002; Walsh \& McGoldrick, 2004).

"A antecipação da perda numa doença física pode ser tão desafiadora e dolorosa para as famílias, como a própria morte de um membro da família” (Rolland 1990, p. 229). Deste modo, perante o diagnóstico de uma doença ameaçadora da vida, e durante todo o curso da mesma, doente e familiares tendem a experimentar um processo de luto antecipatório (Guldin, Vedsted, Zachariae, Olesen, \& Jensen, 2012; Rolland, 1990), cuja expressão tende a exacerbar-se aquando da progressão visível da doença ou aquando da comunicação de más notícias relacionadas com o prognóstico do doente (Lichtenthal, Prigerson, \& Kissane, 2010).

Do ponto de vista sistémico, o luto antecipatório, enquanto processo qualitativamente distinto do luto convencional ou post mortem, implica a compatibilização de 
dois movimentos de reestruturação familiar. Por um lado, a família deve, por ensaio e experimentação, redistribuir os papéis e funções que, anteriormente, pertenciam ao doente terminal, pelos restantes elementos do sistema familiar. Para o efeito, é facilitador que as fronteiras entre os subsistemas sejam flexíveis já que padrões de funcionamento familiar rígidos tendem a bloquear a negociação de novas funções no interior do sistema. Por outro lado, deve estabelecer-se uma nova organização na vida familiar focada no presente e, por conseguinte, no acompanhamento do doente até à sua morte (Relvas, 1989).

O luto antecipatório é, assim, entendido como uma forma de a família, ao antecipar a perda do seu membro, se preparar para trabalhar o trauma da morte e o seu ajustamento após a ocorrência da mesma (Nielsen, Neergaard, Jensen, Bro, \& Guldin, 2016). Algumas respostas, associadas à experiência de luto antecipatório, são comuns e, na maioria das vezes, devem ser entendidas como normativas. Por exemplo, os familiares podem manifestar medo de abandono, ansiedade de separação, sensação de desespero e desamparo, solidão, tristeza, ressentimento, exaustão, culpa (Nielsen et al., 2016; Rolland, 1990), negação, hostilidade e evitamento (Lichtenthal et al., 2010). À medida que a família aceita e se adapta às múltiplas perdas decorrentes da doença, torna-se mais coesa, os seus membros aproximam-se e o vínculo ao familiar doente intensifica-se: despedem-se, manifestam o seu afeto e resolvem eventuais assuntos inacabados com o doente (Lichtenthal et al., 2010; Nielsen et al., 2016; Rolland, 1990).

Concluímos que a eminência da morte deve ser entendida numa perspetiva familiar, uma vez que esta se assume como um dos desafios mais dolorosos para toda a família. Enquanto processo transacional, a experiência do luto antecipatório de um elemento do sistema, repercute-se na dos restantes e vice-versa. Por isso, a resposta familiar à ameaça de perda constitui uma área de estudo e investigação complexa. Porém, importa desenvolver o conhecimento acerca da adaptação dos sistemas familiares à perda, para que as famílias possam ser ajudadas a aceitar a morte como parte integrante da vida e encará-la como um evento de vida transformador, do qual podem decorrer novas possibilidades de crescimento familiar e pessoal (Kissane \& Bloch, 2002; Walsh \& McGoldrick, 2004).

\section{O CUIDADOR PRINCIPAL}

Nas últimas décadas, as mudanças do sistema de saúde têm contribuído para um aumento do acompanhamento dos doentes terminais em situação de ambulatório, isto é, para um aumento das situações em que a prestação de cuidados é feita 
no domicílio, pela família (Campen, Boer, \& Iedema, 2013), cujo papel é central na logística e coordenação dos cuidados. Estes são, geralmente, muito exigentes, requerendo das famílias um vasto conhecimento do tipo de cuidados a prestar, como, quando e onde (Given et al., 2012).

Perante uma doença incapacitante e ameaçadora de vida, há necessidade de destacar um elemento - o cuidador (informal) principal - pertencente, na maioria das vezes, ao sistema familiar, que assume a maior parte dos cuidados ao doente, prestando suporte quer em contexto domiciliário, quer em contexto hospitalar (Carduff et al., 2016).

Devido à exigência na prestação de cuidados e a outros fatores de stress concorrentes, os familiares cuidadores são um grupo particularmente vulnerável, ao desenvolvimento de síndrome de burnout, sintomatologia psicopatológica e níveis preocupantes de distress (Carduff et al., 2016). Podem, ainda, sentir-se isolados e experimentar uma sensação de abandono por parte da família, amigos e outros elementos da rede. Dada a exaustão e sobrecarga, alguns cuidadores podem experimentar o desejo de morte do doente, o que pode provocar, naturalmente, sentimentos de culpa (Wittenberg-Lyles, Demiris, Oliver, \& Burt 2011).

Só respondendo às necessidades experimentadas pelos cuidadores, será possível prevenir respostas desajustadas associadas à doença e prestação de cuidados. Da revisão de literatura, Stajduhar e Cohen (2009) concluíram que estas podem dividir-se em necessidades físicas (e.g., ajuda prática nas tarefas associadas à prestação de cuidados), psicossociais (e.g., prevenção de desenvolvimento de complicações psiquiátricas), cognitivas (e.g., ajuda no planeamento de tarefas complexas, tais como organizar a medicação), financeiras (e.g., ajuda financeira para a compra da medicação) e de suporte (in)formal (e.g., comunicação eficaz com os profissionais de saúde, família e amigos).

Num estudo conduzido por Ewing e Grande (2012), os autores concluíram que as necessidades de suporte experimentadas pelos cuidadores se dividiam em dois grupos de necessidades: a) o suporte relacionado com a prestação de cuidados informais (e.g., informação sobre controlo de sintomas e administração da medicação, informação sobre o processo de morte) e b) o suporte relacionado com o próprio cuidador (e.g., intervalos relativamente à prestação de cuidados, ajuda prática e suporte emocional e/ou espiritual).

Contudo, é relevante salientar que muitos cuidadores conseguem retirar ganhos positivos do acompanhamento do doente terminal (e.g., atribuição de maior sentido à vida). No entanto, os ganhos experimentados pelos cuidadores familiares não são, de modo algum, significado de ausência de efeitos adversos (Totman et al., 2015). Por isso, enquanto elementos críticos na prestação de cuidados ao doente, cuidadores (e restantes familiares) devem ser alvos de um suporte formal ade- 
quado às suas necessidades, com o propósito de poderem retirar, o mais possível, os benefícios que o acompanhamento ao familiar em fim de vida pode oferecer (Stajduhar \& Cohen, 2009).

\section{OS CUIDADOS PALIATIVOS}

A World Health Organization (WHO) (2002) define, os cuidados paliativos como uma

abordagem que visa melhorar a qualidade de vida dos doentes e suas famílias que enfrentam problemas decorrentes de uma doença incurável e/ou grave e com prognóstico limitado, através da prevenção e alívio do sofrimento, com recurso à identificação precoce e tratamento rigoroso dos problemas não só físicos, como a dor, mas também dos psicossociais e espirituais. (p. 84)

Por outras palavras, os cuidados paliativos constituem uma resposta global e multidisciplinar, direcionada aos doentes com patologias que implicam risco de vida como, por exemplo, as oncológicas, do sistema circulatório (e.g., acidente vascular cerebral), respiratório (e.g., doença pulmonar obstrutiva crónica) e nervoso (e.g., doença do neurónio motor) e SIDA/HIV (Keeley \& Noble, 2011). A WHO (2002, 2014) preconiza, ainda, alguns objetivos desta abordagem de cuidados, tais como: prevenir e aliviar a dor ou outras fontes de sofrimento, afirmar a vida e encarar a morte como um processo natural, não antecipar ou protelar intencionalmente a morte, oferecer suporte psicológico e espiritual ao doente, ajudar o doente a viver tão ativo quanto possível até à sua morte, manter a qualidade de vida do doente e oferecer suporte ao sistema familiar quer antes quer após a ocorrência da morte.

Os cuidados paliativos devem ser implementados tão cedo quanto possível e em qualquer fase da doença (e.g., crise, crónica, terminal) com prognóstico fatal (WHO, 2002 , 2014). Este princípio assenta no reconhecimento de que a maioria dos sintomas mais difíceis de gerir não ocorre, apenas, na fase terminal e/ou agónica. Estes sintomas têm a sua origem em fases anteriores da doença e, quando não tratados precocemente, tornam-se difíceis de gerir em fim de vida (Keeley \& Noble, 2011). Com a progressão da doença e sem resposta à terapêutica administrada, os cuidados paliativos tornam-se a abordagem única de prestação de cuidados. No entanto, e especialmente nesta fase, ao contrário dos cuidados curativos que visam curar e modificar o curso da doença, os cuidados paliativos visam capacitar e promover nos doentes e suas famílias todo o seu potencial e qualidade de vida, intervir no 
sofrimento decorrente da doença e responder às necessidades experimentadas pelos doentes e famílias (Irwin \& Ferris, 2008).

Em suma, os cuidados paliativos assumem-se como uma abordagem não curativa de cuidados, que visa prestar cuidados de conforto ao doente, fomentando o seu bem-estar e a melhor qualidade de vida possível até ao seu falecimento, com recurso a soluções tecnicamente corretas e humanamente elevadas (Osswald, 2013). Estes cuidados não se esgotam no acompanhamento ao doente. Cuidar da família em cuidados paliativos constitui um imperativo ético (Bausewein et al., 2015; Osswald, 2013), devendo prestar-se cuidados de conforto, suporte emocional e apoio espiritual quer antes, quer após a ocorrência da morte (Keeley \& Noble, 2011; WHO, 2002, 2014).

\section{O suporte às famílias em cuidados paliativos}

$\mathrm{Na}$ fase terminal da doença, e em cuidados paliativos, a intervenção junto das famílias é tão importante quanto junto do doente (Kristjanson \& Aoun, 2004). Apesar de muitas famílias apresentarem uma resiliência notória na adaptação à fase terminal da doença (Gaudio, Zaider, Brier, \& Kissane, 2011) e retirarem benefícios pessoais enquanto cuidadoras do doente (Totman et al., 2015), outras apresentam níveis de distress significativos (Gaudio et al., 2011), com franca morbilidade psiquiátrica (Askari et al., 2012; Totman et al., 2015). Ainda assim, são muitas as necessidades dos familiares que continuam a não ter resposta por parte dos profissionais de saúde. $\mathrm{O}$ suporte às famílias continua a ser insuficiente e são muito escassos os programas de intervenção validados e cujo desenho seja exequível quer em contexto de internamento, quer em contexto domiciliário (Carduff et al., 2016; Kristjanson \& Aoun, 2004).

As famílias devem ser ajudadas a manter a sua dignidade, paz de espírito, conforto e esperança. Devem promover-se estratégias de coping familiar, preparando-as para a morte, oferecendo um espaço para a ventilação de sentimentos e medos, explorando a narrativa do sentido da vida e da morte (Irwin \& Ferris, 2008). Devem, ainda, ser potenciadas as forças familiares, um funcional ajustamento à doença e o restabelecimento do equilíbrio familiar, fomentando uma comunicação eficaz, um envolvimento colaborativo no processo de tomada de decisão (King \& Quill, 2006), a coesão na unidade familiar e a preservação de relações com outros elementos da rede social (Irwin \& Ferris, 2008).

No que toca aos familiares cuidadores, Milne e Quinn (2009) sintetizam os principais objetivos que a intervenção deve ter. Os objetivos dividem-se em cinco dimensões: informação (e.g., prestar informação sobre a doença), formação 
em competências práticas (e.g., esclarecer como executar as tarefas associadas à prestação de cuidados), suporte social (e.g., promover o contacto com outros cuidadores ou outros elementos da rede pessoal), saúde pessoal (e.g., fomentar a realização de check-ups médicos) e suporte emocional (e.g., trabalhar os medos relativos à morte do doente).

Repare-se que o suporte à família em cuidados paliativos não deve terminar, por definição, com a morte do doente. Estes cuidados devem continuar com o apoio no luto aos familiares (WHO, 2002, 2014). No entanto, não existe consenso no que diz respeito à melhor forma de intervir junto dos familiares enlutados bem como às reais necessidades de suporte que experimentam (Walsh et al., 2007). Do mesmo modo, pouco se sabe sobre os preditores eventualmente associados ao desenvolvimento de luto complicado, pré e pós-morte, num contexto de doença crónica terminal (Nielsen et al., 2016).

\section{A terapia familiar focada no luto}

A Terapia Familiar Focada no Luto (TFFL; Family Focused Grief Therapy), proposta por Kissane e Bloch (2002), assenta numa perspetiva sistémica e familiar e está devidamente validada, através de estudos de eficácia que comprovam a pertinência da sua aplicação junto das famílias em cuidados paliativos (Kissane et al., 2006).

A TFFL assenta na premissa de que a disfunção na família pode estar relacionada a um maior risco de morbilidade psiquiátrica e de desenvolvimento de luto complicado. Por isso, enquanto abordagem profilática, a TFFL é proposta apenas às famílias que apresentem um padrão de funcionamento desajustado (Kissane \& Bloch, 2002; Kissane et al., 2006; Schuler, Zaider, \& Kissane, 2012).

A TFFL tem como objetivos a implementação de estratégias que visem aumentar a coesão, melhorar a comunicação, mobilizar a família para a resolução de conflitos e reforçar as forças da família. Inicia-se, ainda, na fase dos cuidados ao doente terminal e estende-se após a sua morte. É uma abordagem terapêutica breve, focal e limitada no tempo (4-8 sessões; 9-18 meses) e participam nela os membros da família e, em vida (se exequível), o doente (Kissane \& Bloch, 2002; Kissane et al., 2006; Schuler et al., 2012).

Os estudos mostram que esta modalidade de intervenção se revela profícua, pois, ao basear-se numa perspetiva sistémica e familiar, consegue alcançar um maior número de indivíduos em risco psicossocial; reduzir, eficazmente, níveis de distress individual e familiar; e prevenir o desenvolvimento de lutos complicados após a ocorrência da morte (Kissane et al., 2006). 


\section{DISCUSSÃO/CONCLUSÃO}

Cicely Saunders postulava que "o modo como as pessoas morrem, permanece na memória dos que vivem / "how people die remains in the memory of those who live on" (Nurunnabi, 2011, p. 202). Por isso, numa fase tão delicada como a da aproximação da morte de um familiar, a família, conforme preconizado pela WHO (2002, 2014), deve beneficiar de um suporte adequado às necessidades que experimenta (Monroe \& Oliviere, 2009). Acreditamos que, em cuidados paliativos, o suporte às famílias deve ser idealmente multidimensional, integrando uma componente educativa, médica, psicossocial e espiritual.

Reconhecemos, porém, que num contexto de profunda crise económica, os recursos sejam escassos. Deste modo, uma adequada triagem das famílias em maior necessidade de suporte, como proposto por Kissane e Bloch (2002), pode ser muito vantajosa. Sabemos que muitas famílias se adaptam, ao seu ritmo, às exigências que a fase terminal e morte impõem no sistema familiar. Outras, porém, caracterizadas por um funcionamento menos adaptativo (Kissane \& Bloch, 2002) e/ou com outros fatores de risco concorrentes, mostram maiores dificuldades em se ajustarem àquela que é a crise, considerada por alguns autores, mais difícil para o sistema familiar: a doença e a morte (Pereira, 2010; Rolland, 1990, 2005; Walsh \& McGoldrick, 2004).

Todavia, depreendemos com este estudo de revisão de literatura, que a produção empírica sustentada numa perspetiva sistémica é, atualmente, escassa (Carolan et al., 2015). A pouca literatura existente, ainda que basilar, é, na sua maioria, anterior à última década, podendo não refletir fielmente a experiência das famílias de hoje em dia. Por isso, pouco se sabe sobre a resposta familiar à doença e à morte o que, por um lado, nos parece natural, atendendo aos desafios metodológicos e éticos que a investigação empírica com famílias, neste contexto de grande vulnerabilidade, coloca. Além disso, na literatura a que pudemos ter acesso, verificámos que, até à data, e à exceção da Terapia Familiar Focada no Luto (Kissane \& Bloch, 2002), são inexistentes modalidades de intervenção familiar, devidamente validadas através de estudos de eficácia que comprovem a sua adequabilidade e sensibilidade aos problemas emergentes no seio da família, com a aproximação da morte de um dos seus membros.

Acresce que a maior parte dos estudos empíricos focam-se na experiência do cuidador principal, naturalmente considerado um elemento vulnerável neste contexto pelas exigências que lhe são impostas (Stajduhar \& Cohen, 2009) ou, mais pontualmente, na experiência individual dos familiares. Por outro lado, dos estudos existentes, a maior parte centra-se na experiência dos familiares de doentes oncológicos ou, mais raramente, de doentes com Alzheimer. Poucos estudos se debruçam sobre o impacto que outros diagnósticos terminais, como a SIDA, a doença pulmonar obstrutiva crónica ou a esclerose lateral amiotrófica, têm no 
sistema familiar. Cremos que cada doença, quer devido à sintomatologia, quer devido às terapêuticas que lhe são direcionadas, pode colocar desafios específicos ao sistema, que também importa conhecer de forma aprofundada.

Acreditamos, por isso, que o estudo da experiência da unidade familiar no contexto de doença crónica terminal e morte é, ainda, muito incipiente, o que compromete, inevitavelmente, um screening adequado e o desenvolvimento de novas modalidades de intervenção sistémica sensíveis às necessidades dos familiares de doentes terminais, independentemente do seu diagnóstico.

Torna-se fundamental aumentar a produção empírica no âmbito da resposta familiar na fase terminal da doença e, por conseguinte, desenvolver um modelo de compreensão atual e passível de ser validado empiricamente. Só assim, será possível criar e incrementar mais diretrizes que capacitem os profissionais de saúde a prestar um apoio holístico e profilático à família sem que sejam descuradas as suas principais necessidades de suporte e, quando necessário, fazer o seu encaminhamento para uma intervenção psicoterapêutica sensível às problemáticas mais comummente emergentes nesta fase crítica da vida familiar.

O desenvolvimento da investigação neste âmbito é particularmente relevante, uma vez que diversos estudos salientam que a falta de uma abordagem profissional das necessidades e dificuldades das famílias pode ter um impacto altamente negativo no sistema. Pode comprometer quer a vivência funcional e adaptativa da fase terminal da doença (Irwin \& Ferris, 2008) quer a qualidade de vida do próprio doente, ou ainda a vivência normativa do processo de luto pós-morte por parte dos restantes familiares após a ocorrência da morte (Kristjanson \& Aoun, 2004), o que coloca, naturalmente, o sistema em risco de dissolução (Walsh \& McGoldrick, 2004).

Importa ainda salientar uma importante limitação deste estudo que está, fundamentalmente, relacionada com o seu desenho metodológico. Este estudo representa uma revisão não exaustiva da literatura que, por conseguinte, não obedeceu a um método criterioso e sistemático de pesquisa e análise de informação (e.g., critérios de inclusão/exclusão de estudos empíricos). A não padronização dos métodos de recolha e análise dos dados compromete a sua análise e interpretação o que, naturalmente, esteve sujeita à nossa subjetividade. No entanto, e apesar da limitação metodológica apontada, este estudo permitiu a contextualização e o mapeamento da distribuição da produção empírica na área dos cuidados paliativos e família que se depreende, ainda, incipiente.

Assim, considerando a escassez de produção empírica sobre o impacto da doença terminal na família, assente numa perspetiva sistémica e familiar, já atrás referido, consideramos um ponto forte desta revisão de literatura a análise, leitura e interpretação dos resultados de estudos com base nessa perspetiva em detrimento de uma abordagem focada no indivíduo. 
Uma vez que a doença acontece no sistema familiar, com impacto nos seus elementos e relações (Forbat et al., 2012; Kissane \& Bloch, 2002; Rolland, 2005), a intervenção em cuidados paliativos, quando necessária, não se deve esgotar apenas no doente e/ou no cuidador principal. Deve, antes, estender-se a toda a família.

\section{REFERÊNCIAS}

Askari, A., Madgaonkar, J., \& Rowell, R. (2012). Current psycho-pathological issues among partners of cancer patients. Journal of Psychosocial Research, 7(1), 77-85.

Bausewein, C., Simon, S., Pralong, A., Radbruch, L., Nauck, F., \& Voltz, R. (2015). Clinical practice guideline: Palliative care of adult patients with cancer. Deusches Ärzteblatt International, 112(50), 863-870. doi: 10.3238/arztebl.2015.0863

Campen, C., Boer, A., \& Iedema, J. (2013). Are informal caregivers less happy than noncaregivers? Happiness and the intensity of caregiving in combination with paid and voluntary work. Scandinavian Journal of Caring Sciences, 27, 44-50. doi: 10.1111/j.1471-6712.2012.00998.x

Carduff, E., Jarvis, A., Highet, G., Finucane, A., Kendall, M., Harrison, N., ... Murray, S. (2016). Piloting a new approach in primary care to identify, assess and support carers of people with terminal illnesses: A feasibility study. BMC Family Practice, 17(16), 1-9. doi: 10.1186/s12875-016-0414-2

Carolan, C., Smith, A., \& Forbat, L. (2015). Conceptualising psychological distress in families in palliative care: Findings from a systematic review. Palliative Medicine, 29(7), 605-632. doi: $10.1177 / 0269216315575680$

Ewing, G., \& Grande, G. (2012). Development of a Carer Support Needs Assessment Tool (CSNAT) for end-of-life care practice at home: A qualitative study. Palliative Medicine, 27(3), 244-256. doi: $10.1177 / 0269216312440607$

Forbat, L., McManus, E., \& Haraldsdottir, E. (2012). Clinical implications for supporting caregivers at the end-of-life: Findings and from a qualitative study. Contemporary Family Therapy, 34(2), 282-292. doi: 10.1007/s10591-012-9194-6

Gaudio, F., Zaider, T., Brier, M., \& Kissane, D. (2011). Challenges in providing family-centered support to families in palliative care. Palliative Medicine, 26(8), 1025-1033. doi: $10.1177 / 0269216311426919$

Given, B., Given, C., \& Sherwood, P. (2012). Family and caregiver needs over the course of the cancer trajectory. The Journal of Supportive Oncology, 10(2), 57-63. doi: 10.1016/j.suponc.2011.10.003

Gonçalves, J., Alvarenga, M., \& Silva, A. (2003). The last forty-eight hours in a Portuguese palliative care unit: Does it differ from elsewhere? Journal of Palliative Medicine, 6(6), 895-900.

Guldin, M., Vedsted, P., Zachariae, F., Olesen, F., \& Jensen, A. (2012). Complicated grief and need for professional support in family caregivers of cancer patients in palliative care: A longitudinal cohort study. Supportive Care in Cancer, 20(8), 1679-1685. doi: 10.1007/ s00520-011-1260-3

Irwin, S., \& Ferris, F. (2008). The opportunity for psychiatry in palliative care. Canadian Journal of Psychiatry, 53(11), 713-724.

Keeley, P., \& Noble, S. (2011). Palliative care: Introduction. Medicine, 39(11), 635. doi: 10.1016/j. mpmed.2011.08.005 
King, D., \& Quill, T. (2006). Working with families in palliative care: One size does not fit all. Journal of Palliative Medicine, 9(3), 704-715. doi:10.1089/jpm.2006.9.704

Kissane, D., \& Bloch, S. (2002). Family Focused Grief Therapy: A model of family-centered care during palliative care and bereavement. London, England: Open University Press.

Kissane, D., McKenzie, M., Bloch, S., Moskowitz, C., McKenzie, D., \& O’Neill, I. (2006). Family focused grief therapy: A randomized, controlled trial in palliative care and bereavement. The American Journal of Psychiatry, 163(7), 1208-1218. Consultado em http://ajp.psychiatryonline.org/doi/ full/10.1176/ajp.2006.163.7.1208

Kristjanson, L., \& Aoun, S. (2004). Palliative care for families: Remembering the hidden patients. Canadian Journal of Psychiatry, 49(6), 359-365.

Lichtenthal, W., Prigerson, H., \& Kissane, D. (2010). Bereavement: A special issue in oncology. In J. Holland, W. Breitbart, P. Jacobsen, M. Lederberg, M. Loscalzo, \& R. McCorkle (Eds.), PsychoOncology (2 ${ }^{\text {nd }}$ ed., pp. 537-543). New York: Oxford University Press.

Milne, D., \& Quinn, K. (2009). Family carers of people with advanced cancer. In P. Hudson, \& S. Payne (Eds.). Family carers in palliative care. A guide for health and social care professionals. (pp. 211-230). New York: Oxford University Press.

Monroe, B., \& Oliviere, D. (2009). Communicating with family carers. In P. Hudson \& S. Payne (Eds.), Family carers in palliative care: A guide for health and social care professionals (pp. 1-20). New York: Oxford University Press.

Nielsen, M., Neergaard, M, Jensen, A., Bro, F., Guldin, M. (2016). Do we need to change our understanding of anticipatory grief in caregivers? A systematic review of caregiver studies during end-of-life caregiving and bereavement. Clinical Psychology Review, 44, 75-93. doi: 10.1016/j. cpr.2016.01.002

Nurunnabi, A. (2011). Palliative care ethics. Journal of Dhaka Medical College, 20(1), 199-203. doi: 10.3329/jdmc.v20i2.10550

Osswald, W. (2013). Sobre a morte e o morrer. Lisboa: Fundação Francisco Manuel dos Santos.

Pereira, S. (2010). Cuidados paliativos: Confrontar a morte. Lisboa: Universidade Católica Editora.

Relvas, A. (1989). Morte e luto na família: Uma abordagem sistémica. Psicologia Clínica, 1, 49-59.

Rolland, J. (1990). Anticipatory loss: A family systems developmental framework. Family Process, 29(3), 229-244.

Rolland, J. (2003). Mastering family challenges in serious illness and disability. In F. Walsh (Ed.), Normal family processes: Growing diversity and complexity ( $3^{\text {rd }}$ ed., pp. 460-489). New York, NY: The Guilford Press.

Rolland, J. (2005). Cancer and the family: An integrative model. Cancer, 104(S11), 2584-2595. doi: $10.1002 /$ cncr.21489

Schuler, T., Zaider, T., \& Kissane, D. (2012). Family grief therapy: A vital model in oncology, palliative care and bereavement. Family Matters, 90(1), 77-86.

Sociedad Española de Cuidados Paliativos (s.d.). Guía de Cuidados Paliativos. Consultado em http:// www.secpal.com/\%5CDocumentos\%5CPaginas\%5Cguiacp.pdf

Stajduhar, K., \& Cohen, R. (2009). Family caregiving in the home. In P. Hudson \& S. Payne (Eds.), Family carers in palliative care: A guide for health and social care professionals (pp. 149-168). New York: Oxford University Press.

Totman, J., Pistrang, N., Smith, S., Hennessey, S., \& Martin, J. (2015). 'You only have one chance to get it right': A qualitative study of relatives' experiences of caring at home for a family member with terminal cancer. Palliative Medicine, 29(6), 96-507. doi: 10.1177/0269216314566840 
Wadhwa, D., Burman, D., Swami, N., Rodin, G., Lo, C., \& Zimmermann, C. (2013). Quality of life and mental health in caregivers of outpatients with advanced cancer. Psycho-Oncology, 22(2), 403-410. doi: 10.1002/pon.2104

Walsh, F. (2002). A family resilience framework: Innovative practice applications. Family Relations, 51(2), 130-137. doi: 10.1111/j.1741-3729.2002.00130.x

Walsh, F. (2003). Family resilience: Strengths forged through adversity. In F. Walsh (Ed.), Normal family processes: Growing diversity and complexity ( ${ }^{\text {rd }}$ ed., pp. 399-423). New York, NY: The Guilford Press.

Walsh, F., \& McGoldrick (2004). Loss and the family: A systemic perspective. In F. Walsh \& M. McGoldrick (Eds.). Living beyond the loss: Death in the family ( $2^{\text {nd }}$ ed., pp. 3-26). New York: W. W. Norton \& Company.

Walsh, K., Jones, L., Tookman, A., Mason, C., Mcloughlin, J., Blizard, R., \& King, M. (2007). Reducing emotional distress in people caring for patients receiving specialist palliative care. British Journal of Psychiatry, 190, 142-147. doi: 10.1192/bjp.bp.106.023960

Wittenberg-Lyles, E., Demiris, G., Oliver, D., \& Burt, S. (2011). Reciprocal suffering: Caregiver concerns during hospice care. Journal of Pain and Symptom Management, 41(2), 383-393. doi: 10.1016/j.jpainsymman.2010.04.026

World Health Organization (2002). National cancer control programmes: Policies and managerial (2 $2^{\text {nd }}$ ed.). Geneva: World Health Organization.

World Health Organization (2014). Global atlas of palliative care at the end of life. London: Worldwide Palliative Care Alliance. 